\title{
Forest Fire Prevention Early Warning Method Based on Fuzzy Bayesian Network
}

\author{
Jun Lin ${ }^{1}$, Hanjiang Dong ${ }^{1}$, Wenxiao Liu ${ }^{1}$, Junlong $\mathrm{Li}^{1}$, Haitian $\mathrm{Lu}^{1}$, Kuncheng Ou ${ }^{1}$, Dongsong Yan $^{1}$, Wenchao Lian ${ }^{1}$ \\ ${ }^{1}$ School of Electrical Engineering, Jinan University, Zhuhai, China \\ Correspondence: Dongsong Yan, School of Electrical Engineering, Jinan University, No. 206, Qianshan Road, \\ Xiangzhou District, Zhuhai City, Guangdong Province, China.
}

Received: March 20, 2019

doi:10.11114/set.v6i1.4161
Accepted: April 23, 2019 Online Published: April 27, 2019

URL: https://doi.org/10.11114/set.v6i1.4161

\begin{abstract}
In the environment of large forest, the factors causing fire are nonlinear and uncertain. If the data collected by the sensor is simply analyzed and compared, the false alarm rate will be higher. How to combine the data of several sensors for effective fire warning is a difficult point. In order to improve the accuracy of prediction, aiming at the shortcomings of traditional forest fire prevention early warning system, we propose a forest fire prevention early warning method based on fuzzy Bayesian network. Firstly, we combine the fuzzy control system and the Bayesian network in series, and pre-process the collected sensor data. The pre-processed data is sent to the previously trained Bayesian network for processing. Then the calculated open fire probability, smoldering fire probability, and no fire probability are used as input data of fuzzy control system, and fuzzy inference is performed. Finally, we de-fuzzify the results of fuzzy reasoning and get the probability of fire. Simulation results show that our method can effectively combine the data collected by multiple sensors, quickly and accurately determine fire occurrence probability, improve the accuracy of forest fire prevention warning, and reduce the false positive rate.
\end{abstract}

Keywords: forest fire prevention warning, Bayesian network, fuzzy system

\section{Introduction}

Forest fires are characterized by sudden bursts, rapid spread, and high hazard, which is difficult to extinguish. How to give the probability of forest fires in a timely and accurate manner and prevent the emergence of fire sources is particularly important. Many of the factors that cause forest fires change irregularly over time, making identification of forest fires very difficult. Using a single sensor or simply analyzing multiple sensor signals to determine the likelihood of a fire can result in a higher false alarm rate and false negative rate for the fire. At present, most of the literature is based on the research on the fire size and fire development trend after the fire, and has achieved scientific research results, but there are few studies on the algorithm for calculating the probability of fire occurrence. Shijun Sun (Shijun Sun, 2017) proposed a forest fire monitoring and early warning model based on grid GIS platform. Chen Zhang (Chen Zhang, 2017) studied the fire alarm and positioning scheme based on infrared technology. Yajun Liu (Yajun Liu, 2017) introduced a remote sensing fire monitoring model based on meteorological satellites. Dong Wang (Dong Wang, 2017) designed a forest fire management system based on big data. Chunxiang Liu (Chunxiang Liu, 2017) established a transmission line mountain fire risk assessment model based on BP neural network. Different from above methods, we find a new method based on the characteristics of Bayesian network and fuzzy system, which only need to be carried by a drone instead of launching satellites or building GIS platforms.

Bayesian networks and fuzzy control systems have their own basic characteristics and applications. Fuzzy control system is based on people's control experience and knowledge of the controlled system (Jianrong Gu, 2000). It is especially suitable for complex objects that are difficult to model or cannot be modeled. It is easy to be accepted by people, and the algorithm is simple, easy to implement, and extremely strong robustness. Bayesian network based on probabilistic reasoning (Finn Verner Jensen, 1996) is a technology developed in recent years to solve the problem of uncertainty and incompleteness. It is very expert in solving the faults caused by complex system uncertainty and interconnectivity and currently becomes one of the most effective theoretical models in the field of uncertain knowledge representation and reasoning. Similarly, Jiangzhou C proposed a fuzzy cognitive Bayesian network modeling method based on the possibility principle for fault diagnosis of hydropower units (Jiangzhou Cheng, 2018). Therefore, based on 
the characteristics of Bayesian network and fuzzy system, we design a forest fire prevention early warning method model based on fuzzy Bayesian network.

We mainly classify the input sensor detection data into the Bayesian network, then send the Bayesian network output signal to the fuzzy control system for fuzzy reasoning, and finally output the fire probability and make a judgment of forest fire. The biggest feature of this method is that the Bayesian network and the fuzzy control system are independent of each other. The Bayesian network first processes the data samples to achieve data collection and aggregation, and can suppress noise and then send it to the fuzzy control system, which makes the acquisition of fuzzy rules becomes easier.

\section{Method}

\subsection{Forest Fire Prevention Warning Model}

There must be three conditions for forest fires: 1. Combustible materials (including trees, grass irrigation, etc.) are the material basis for forest fires; 2 . Weather is an important condition for fires; 3 . Fire sources are the main cause of forest fires. If one of the above three conditions is missing, a forest fire will not occur. Therefore, the temperature, relative humidity, smoke concentration and $\mathrm{CO}$ concentration of the forest are collected by temperature sensor, humidity sensor, smoke sensor and $\mathrm{CO}$ concentration sensor respectively, and the fire warning analysis is carried out in combination with the number of consecutive sunny days and the number of flammable plants in the local forest. Our forest fire prevention warning model is shown in figure 1.

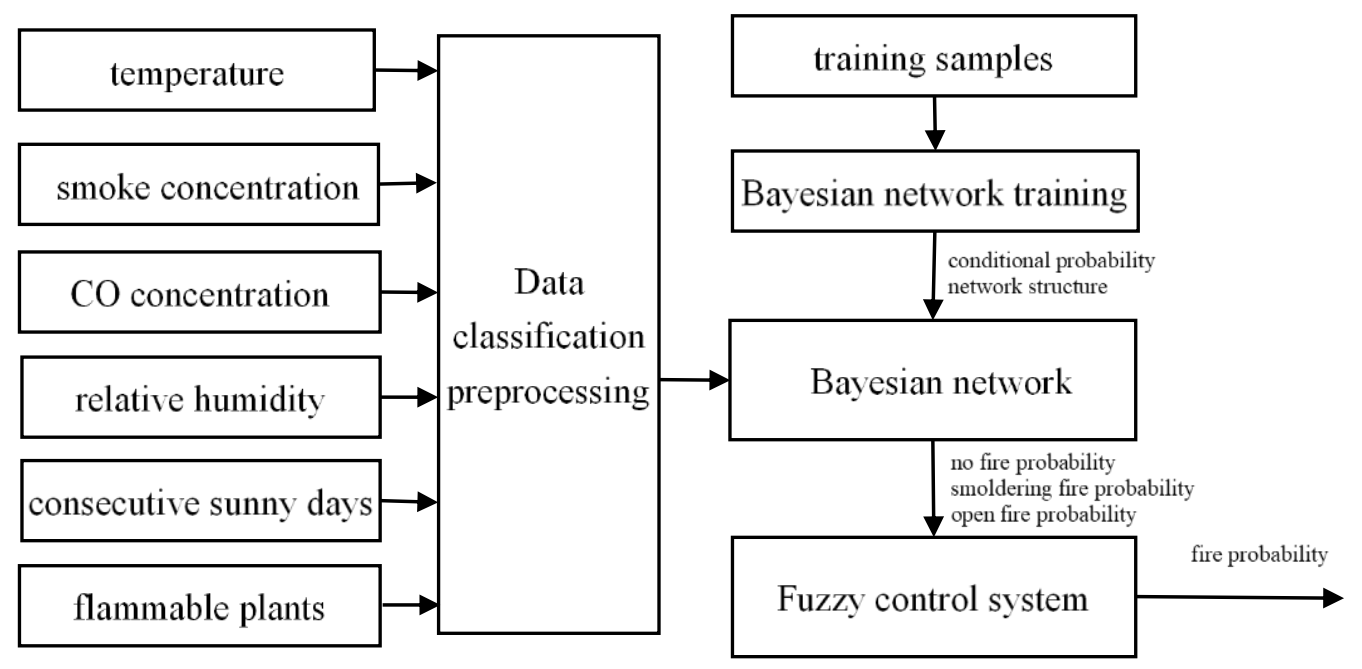

Figure 1. Forest fire prevention warning model

The fuzzy Bayesian network we use is a series structure, which is mainly divided into three parts: 1. The training of Bayesian network. The training sample set is given to obtain the optimal network structure. Then, using the network topology, training sample set and prior knowledge, the conditional probability density at each node of the Bayesian network model is determined, and the Bayesian network training is completed. 2. Use the Bayesian network to predict the probability of fire. Firstly, the data collected by temperature sensor, humidity sensor, smoke sensor and gas sensor, as well as the number of local continuous sunny days and the number of flammable plants is classified and processed as the input vector of the Bayesian network. The data is processed by the Bayesian network and output open fire probability, smoldering fire probability and no fire probability. 3. Calculate the probability of the final fire. Firstly, open fire probability, smoldering fire probability and no fire probability are sent to the fuzzy system, then the appropriate membership function is selected to fuzzify the input probability value, the fuzzy rule is established, then the fuzzy reasoning is performed, and finally the defuzzification is performed.

\subsection{Forest Fire Prevention Early Warning Algorithm Based on Fuzzy Bayesian Network}

\subsubsection{Data Classification Preprocessing}

We classify the data collected by temperature sensors, smoke sensors, humidity sensors and gas sensors (Hongyuan Ge, 2018), as well as the number of sunny days and the number of flammable plants, as a secondary feature node of the Bayesian network (Xiaowei Li, 2013). Value of the feature node is divided into four levels: general, high, high, and very high, and the values are 0/1/2/3/4 respectively. Then we train the Bayesian network structure, the combustion nodes are graded, and the open fire probability is divided into four levels: open fire probability (PB), open fire probability (PM), open fire probability (PS), and open fire probability zero (NP) and the values are 0/1/2/3 respectively; the probability of smoldering fire is divided into smoldering fire probability (PB), smoldering fire probability (PM), smoldering fire probability (PS), smoldering fire probability zero (NP) and the values are 0/1/2/3 respectively; the non-fire probability is divided into three levels: no fire 
probability (PB), no fire probability (PM), and no fire probability (PS) and the values are 0/1/2 respectively.

\subsubsection{Bayesian Network Training}

Many of the factors that cause forest fires change irregularly with time (Hongyu Gao, 2018). The data collected by the sensors have large differences (including approximate continuity, large span discreteness), so they need to be built before the Bayesian network is built (Lam Wai, 2002). This process of ranking is complicated. In the process of establishing the Bayesian network model, we use the structure learning algorithm and the EM (expectation-maximization) algorithm with multiple iterations to establish the forest fireproof Bayesian network.

Bayesian network structure learning is to find a network structure that matches the training sample set $D$ given a set of data samples $D$.

Our Bayesian network structure learning algorithm uses the K2 scoring algorithm and uses $P(G, D)$ as the scoring function (Qingsong Cai, 2018):

$$
\begin{aligned}
B(G, D)=P(G, D) & =P(G) / P(D \mid G) \\
& =P(G) \prod_{i=1}^{N} P\left(x_{i} / G\right) \\
& =P(G) \prod_{i=1}^{n} \prod_{j=1}^{q_{i}} \frac{\left(r_{i}-1\right)}{\left(N_{i j}+r_{i}-1\right)} \prod_{k=1}^{r_{i}} N_{i j k} !
\end{aligned}
$$

Where:

$P(G)=$ The prior probability of the network structure $G$

$X_{i}=$ The network node which has status $x_{i}^{1}, x_{i}^{2}, \ldots, x_{i}^{r_{i}}$, that is, $X_{i} \in\left\{x_{i}^{1}, x_{i}^{2}, \ldots, x_{i}^{r_{i}}\right\}$

$\prod i=$ The corresponding parent set of $X_{i}$ whose order is $1,2, \ldots, q_{i}$ with the configuration $\pi_{i}$

$$
N_{i j k}=\prod_{k=1}^{r_{i}} N_{i j k}=\text { the number of cases in which } X_{i}=x_{i}{ }^{k} \text { and } \pi_{i}=j \text { are satisfied in the data set } D
$$

For example, if an expert suggests that there is a particular edge or a local structure, then a given network structure should be given a higher prior probability. If there is no prior probability for the network structure, or there is no special priority network structure, then the prior probability $P(G)$ can be assumed to be uniformly distributed, that is, $P(G)=c$, and $c$ is a constant. Given a network structure $G$, the conditional probability $\theta_{i j k}$ can be estimated by a Bayesian estimator:

$$
\theta_{i j k}=E\left(\theta_{i j k} \mid D, G\right)=\left(N_{i j k}+1\right) /\left(N_{i j}+r_{i}\right)
$$

Where:

\section{$E=$ Expectation}

The Bayesian score can be interpreted as: If for all network structures $G$, a network structure $G 0$ has $P(G 0, D) \geq P(G, D)$, then for the current data set $D, G 0$ is Bayeux. The score is the most consistent with the network structure of $D$. In the algorithm implementation process, the formula (2) is usually simplified, and $\log (P(G, D))$ is used instead of $P(G, D)$ to obtain the decomposition form of the scoring function as follows.

$$
\begin{aligned}
f_{K 2}(G: D) & =\sum_{i}^{n} f_{K 2}\left(x_{i}, \prod\left(x_{i}\right): N_{x_{i} \prod\left(x_{i}\right)}\right) \\
& =\sum_{i=1}^{q_{i}}\left(\log \left(\frac{\left(r_{i}-1\right) !}{\left(N_{i j}+r_{i}-1\right) !}\right)\right)+\sum_{k=1}^{r_{i}} \log \left(N_{i j k} !\right)
\end{aligned}
$$

The parameter learning of Bayesian network seeks the probability distribution of each node of the network based on the sample data. We use the network topology and training sample set and prior knowledge to determine the conditional 
probability density at each node of the Bayesian network model, denoted as $P(\theta \mid D, G)$. The Bayesian network parameter learning algorithm is actually the process of solving the convergence to the optimal parameters of the local nodes $\theta_{s}$. Firstly, the $\theta_{s}$ configuration is initialized, and then the iterative $E$ and $M$ are used to find the maximum posterior probability hypothesis and converge the optimal value. The maximum likelihood estimation of the data is carried out to simulate the parameters that best fit the structure. The specific steps are as follows:

1) $E$ step (Expectation)

$$
\begin{aligned}
& E_{p\left(x \mid D, \theta_{S}, S^{h}\right)}\left(N_{i j k}\right)=\sum_{l=1}^{N} P\left(x_{i}^{k}, \pi_{i}^{j} \mid y_{l}, \bar{\theta}_{S}, S^{h}\right) \\
& h, i, j, k, l, S \in N
\end{aligned}
$$

Where:

$D=$ Training samples

$\bar{\theta}_{S}=$ Optimal parameters, in which $X_{i} \in\left\{x_{i}^{1}, x_{i}^{2}, \ldots, x_{i}^{r_{i}}\right\}$

$N_{i j k}=\prod^{r_{i}} N_{i j k}=$ the number of cases in which $X_{i}=x_{i}{ }^{k}$ and $\pi_{i}=j$ are satisfied in the data set $D$

$y_{i}=$ The humber of data lost in $D$

$S^{h}=$ The Bayesian network structure selection hypothesis

2) $\quad M$ step(Maximum estimate)

We list the maximum likelihood estimation function as follows:

$$
\theta_{i j k}=\frac{E_{p\left(x \mid D, \theta_{S}, S^{h}\right)}\left(N_{i j k}\right)}{\sum_{k=1}^{q_{i}} E_{p\left(x \mid D, \theta_{S}, S^{h}\right)}\left(N_{i j k}\right)}
$$

Maximum posterior estimate is

$$
\begin{aligned}
& \theta_{i j k}=\frac{N_{i j k}^{\prime}+E_{p\left(x \mid D, \theta_{S}, S^{h}\right)}\left(N_{i j k}\right)}{\sum_{k=1}^{q_{i}}\left(N_{i j k}^{\prime}+E_{p\left(x \mid D, \theta_{S}, S^{h}\right)}\left(N_{i j k}\right)\right)} \\
& i, j, k, h, q \in N
\end{aligned}
$$

Where:

$N_{i j k}^{\prime}=$ Prior statistical sufficient factor

$N_{i j k}=$ Sample data sufficient statistical factor

\subsubsection{Fuzzy Control System Design}

The three output signals of the Bayesian network are used as the input of the fuzzy control system, and then the fuzzy logic processing is performed to improve the output precision and fault tolerance of the forest fire prevention early warning system.

The fuzzy rule form is "If open fire probability is $A_{i}$ and smoldering fire probability is $B_{i}$ and no fire probability is $C_{i}$, then fire probability of is $P_{i}$ ". Among them, $A_{i}, B_{i}$ and $C_{i}$ respectively indicate the fuzzy quantization level of open fire probability, smoldering fire probability, and no fire probability, which is the quantitative level of fire probability.

According to the experience of experts in the field of forest fires, and through a large number of experimental experiments, get 48 fuzzy inference rules, such as: if (open fire probability is NP) and (smoldering fire probability is NP) and (no fire probability is PS) then (fire probability is PS); if (open fire probability is NP) and (smoldering fire probability is NP) and (no fire probability is PM) then (fire probability is NP).

Taking the above fuzzy control rule "if $\mathrm{x}$ is $A_{i}$ and $\mathrm{y}$ is $B_{i}$ and $\mathrm{z}$ is $C_{i}$, then $\mathrm{u}$ is $P_{i}$ ", the corresponding fuzzy implication relationship is defined as (Yaqin Zhao, 2015) 


$$
R_{i}=\left(A_{i} \times B_{i} \times C_{i}\right) \times P_{i}
$$

Where:

$$
\begin{aligned}
& x=\text { Open fire probability } \\
& y=\text { Smoldering fire probability } \\
& z=\text { No fire probability } \\
& u=\text { Fire probability }
\end{aligned}
$$

For all fuzzy implication relations, the fuzzy relationship corresponding to all control rules is

$$
R=\bigcup_{i=1}^{48} R_{i}=\bigcup_{i=1}^{48}\left(A_{i} \times B_{i} \times C_{i} \times D_{i}\right)
$$

For the fuzzy control rule is " if $\mathrm{x}$ is $A_{i}$ and $\mathrm{y}$ is $B_{i}$ and $\mathrm{z}$ is $C_{i}$, then $\mathrm{u}$ is $P_{i}$ ", then fuzzy reasoning can be used to find that the fire probability $P_{i}$ is

$$
P_{i}=\left(A \text { and } B^{`} \text { and } C^{`}\right) \circ R
$$

The resulting $P_{i}$ is a fuzzy set that needs to be refined. We use the center of gravity method to accurately process the fire probability to obtain the accurate output of fire probability $u_{0}$ :

$$
\begin{aligned}
\boldsymbol{\mu}_{p_{i}}\left(u_{i}\right)=\vee\left[\wedge A_{i}(x) \wedge B_{i}(y) \wedge C_{i}(z) \wedge R(x, y, z, u)\right] \\
u_{0}=\frac{\sum_{i} \mu_{p_{i}}\left(u_{i}\right) \times u_{i}}{\sum_{i} \mu_{p_{i}}\left(u_{i}\right)}
\end{aligned}
$$

\subsection{An Example of Forest fire Prevention Early Warning Method Based on Fuzzy Bayesian Network}

In this section we build a Bayesian network model for forest fire prevention based on expert knowledge and historical data in the field of forest fire prevention. The Full BNT-1.04 platform is used for simulation research, and the output results of Bayesian network and fuzzy control are simulated and verified. Verify the effectiveness of the method model for forest fire prevention warnings.

\subsubsection{Building a Bayesian Network Model}

In the Bayesian network model, there are three kinds of nodes: (1) first characteristics nodes; (2) secondary characteristics nodes; and (3) burning nodes. The first characteristics nodes are connected to the secondary characteristics nodes, and the secondary characteristics nodes are connected to the burning nodes. figure. 2 is a model diagram of a forest fireproof Bayesian network initially established using the MATLAB aids on the Full BNT-1.04 platform based on expert knowledge and historical data in the field of forest fire prevention.

Table 1. Burning node included in the model

$\begin{array}{cc}\text { Smoldering fire probability } & \text { Q1 } \\ \text { No fire probability } & \text { Q2 } \\ \text { Open fire probability } & \text { Q3 }\end{array}$

Table 2. Feature nodes included in the model

\begin{tabular}{cc}
\hline $\begin{array}{c}\text { Feature level node } \\
\text { Node name }\end{array}$ & Feature secondary node \\
Node name
\end{tabular}




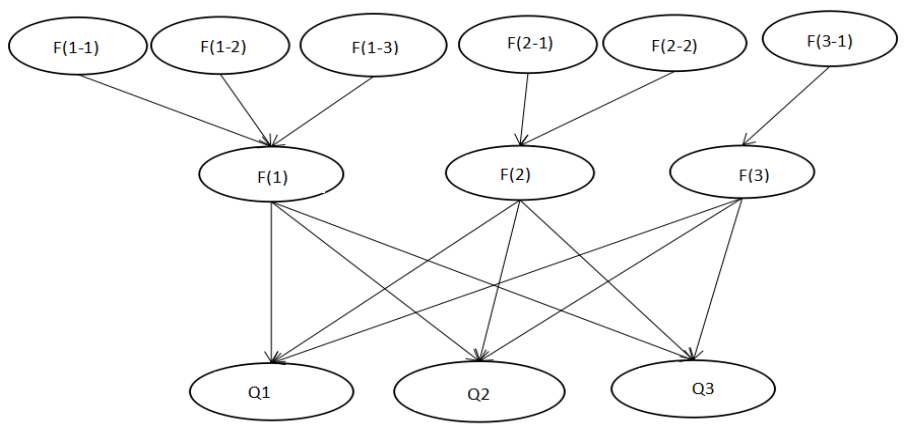

Figure 2. Forest fire prevention warning model

\subsubsection{Bayesian Network Accuracy Verification Experiment}

According to the forest fireproof Bayesian network obtained above, and using this network as the true value, the network uses probability sampling method to generate 6000 sets of sample data, which is divided into 10 experiments, each experimental data is 600 sets, and the first 550 sets are used as training data, the last 50 groups were used as test data. Table 3. lists the training and test data for one of the experiments. Using the structure learning K2 scoring algorithm and the parameter learning $E M$ algorithm given above, 550 sets of training data were used to train the new Bayesian network. Then, the characteristic node data of 50 sets of test data is input into the trained Bayesian network, and the burning node prediction result of 50 sets of data is obtained, and the state of 3 fault nodes of $Q 1$ and $Q 3$ of the original 50 sets of data is compared. Take $Q 2$ as an example. If the predicted number of datas in 50 sets of data is $\mathrm{M}$, and the total number of datas is $\mathrm{N}=50$, the correct rate of $Q 2$ is $P Q 2=M / N$, and the correct rate of the three faulty nodes $Q 1$ to $Q 3$ are respectively counted. We take $Q 2$ (no fire probability) as an example to study and calculate the correct rate. The predicted result statistics of the node $Q 2$ after 10 experiments are shown in Table 4. For other burning nodes, the experimental verification results are similar.

Table 3. The 550 sets of training data and 50 sets of test data for the first experiment

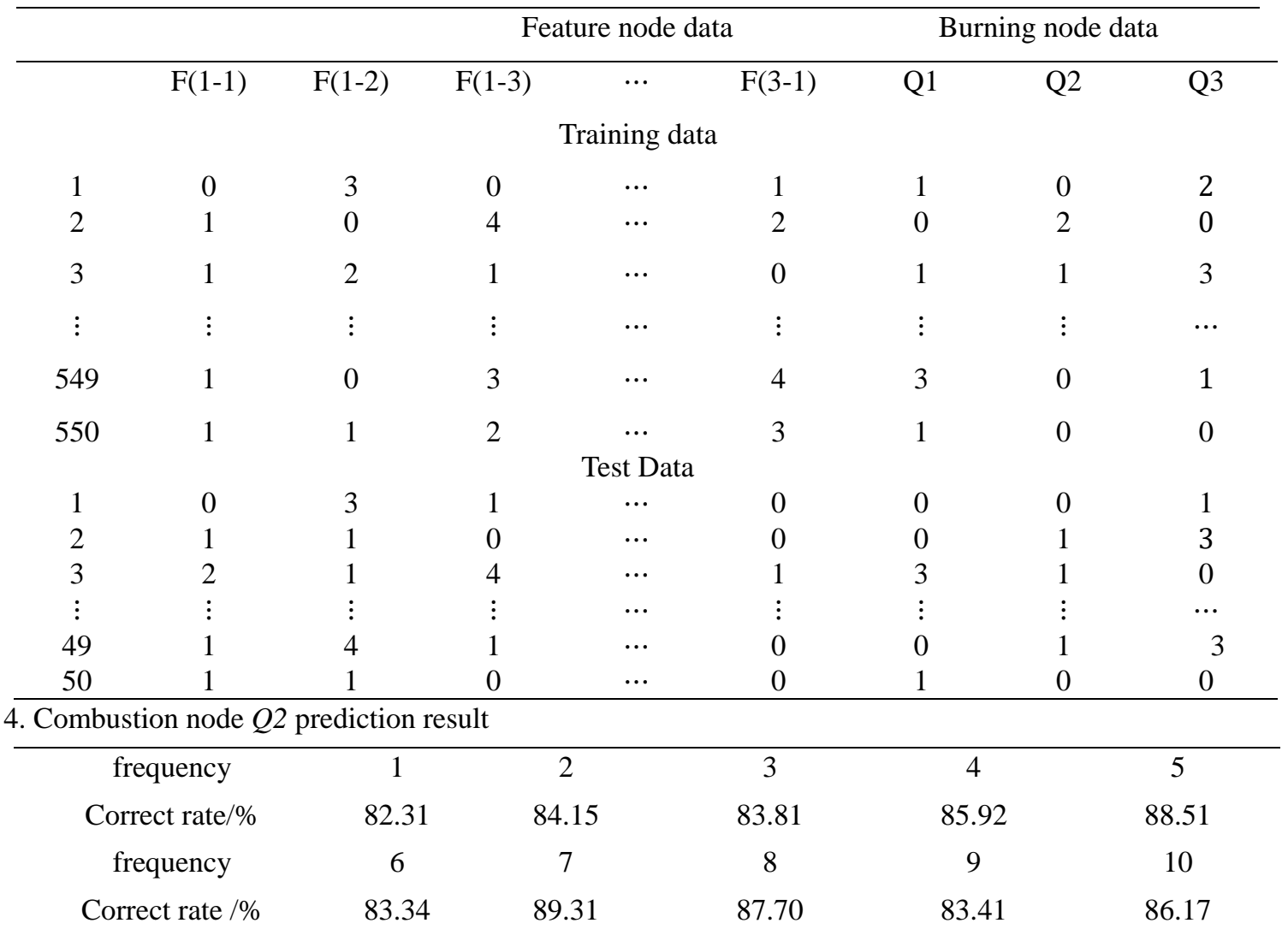

From the data in Table 4, it can be concluded that the average prediction accuracy of 10 experiments is $85.463 \%$. The average training time recorded was $98 \mathrm{~s}$. The Bayesian network after training has a high correct rate and the program runs fast. 


\subsubsection{Different Data Volume Prediction Experiments}

This experiment was conducted to find the amount of training data suitable for the Bayesian network studied in this paper. According to the probability network, 12000 sets of data were generated by the known network, and the experiment was divided into 10 experiments. The data of each experiment was 1200 groups. The first 1150 groups were used as training data, and the last 50 groups were used as test data. The experimental steps are the same as those in Section 4.2. Similarly, one of the three burning nodes $Q 2$ (no fire probability) is selected, and the prediction results of the node $Q 2$ after repeating 10 experiments are shown in Table 5. The average training time recorded was 190s. For other faulty nodes, the experimental verification results are similar.

Table 5. Forecast accuracy table for 1200 sets of data

\begin{tabular}{cccccc}
\hline frequency & 1 & 2 & 3 & 4 & 5 \\
Correct rate/\% & 85.42 & 84.23 & 87.65 & 83.25 & 90.33 \\
frequency & 6 & 7 & 8 & 9 & 10 \\
Correct rate $/ \%$ & 83.69 & 82.24 & 86.13 & 81.33 & 83.32 \\
\hline
\end{tabular}

Then, the known network generates 18000 sets of data according to probability sampling, and the above experiment is repeated. The prediction result of the node Q2 is shown in Table 6. The average training time recorded was 451s.

Table 6. Forecast accuracy table for 1800 sets of data

\begin{tabular}{cccccc}
\hline frequency & 1 & 2 & 3 & 4 & 5 \\
Correct rate/\% & 86.66 & 86.18 & 86.33 & 83.34 & 85.86 \\
frequency & 6 & 7 & 8 & 9 & 10 \\
Correct rate $/ \%$ & 86.42 & 87.11 & 88.35 & 85.19 & 84.94 \\
\hline
\end{tabular}

Draw a line chart according to Table 4 - Table 6 to observe the effect of different numbers of training samples on the correct rate of Bayesian network output.

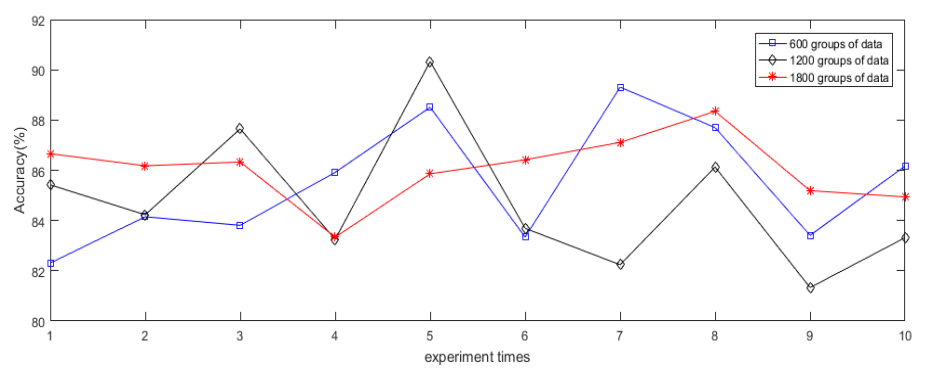

Figure 3. Correct rate of forest fireproof Bayesian network under different data volume training

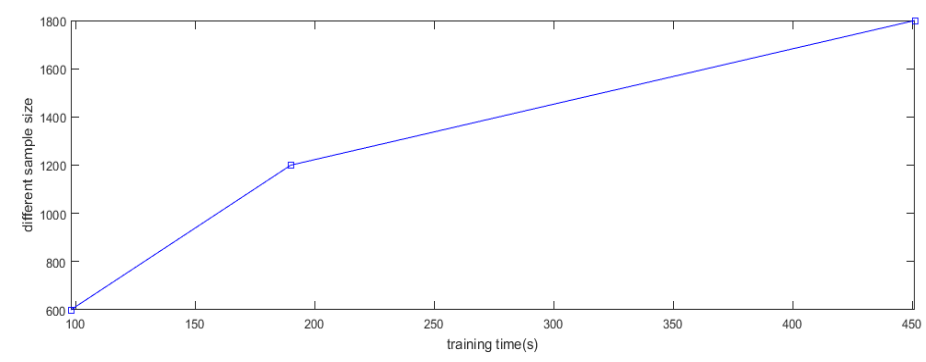

Figure 4. Forest fire prevention Bayesian network training time under different data volumes

As can be seen from figure 3 and figure 4, since the algorithm used in the network learning includes iterative processes, as the amount of data increases, although the accuracy of the Bayesian network increases slightly, the training time increases substantially. It can be seen that the amount of data required for training the Bayesian network is moderate, not as much as possible.

\subsubsection{Fire Warning Performance Verification}

The fuzzy controller outputs the fire probability, which is based on the fuzzy proposed in this paper. The final output of the Bayesian network, as shown in figure 5 and figure 6, shows the relationship between open fire probability and smoldering fire probability, open fire probability and no fire probability, smoldering fire probability and no fire 
probability and fire probability. The simulation results show that the trend of change is relatively smooth, and fire probability increases with the increase of open fire probability and smoldering fire probability, and decreases with the increase of no fire probability. Therefore, the designed fuzzy controller is ideal and meets the requirements.

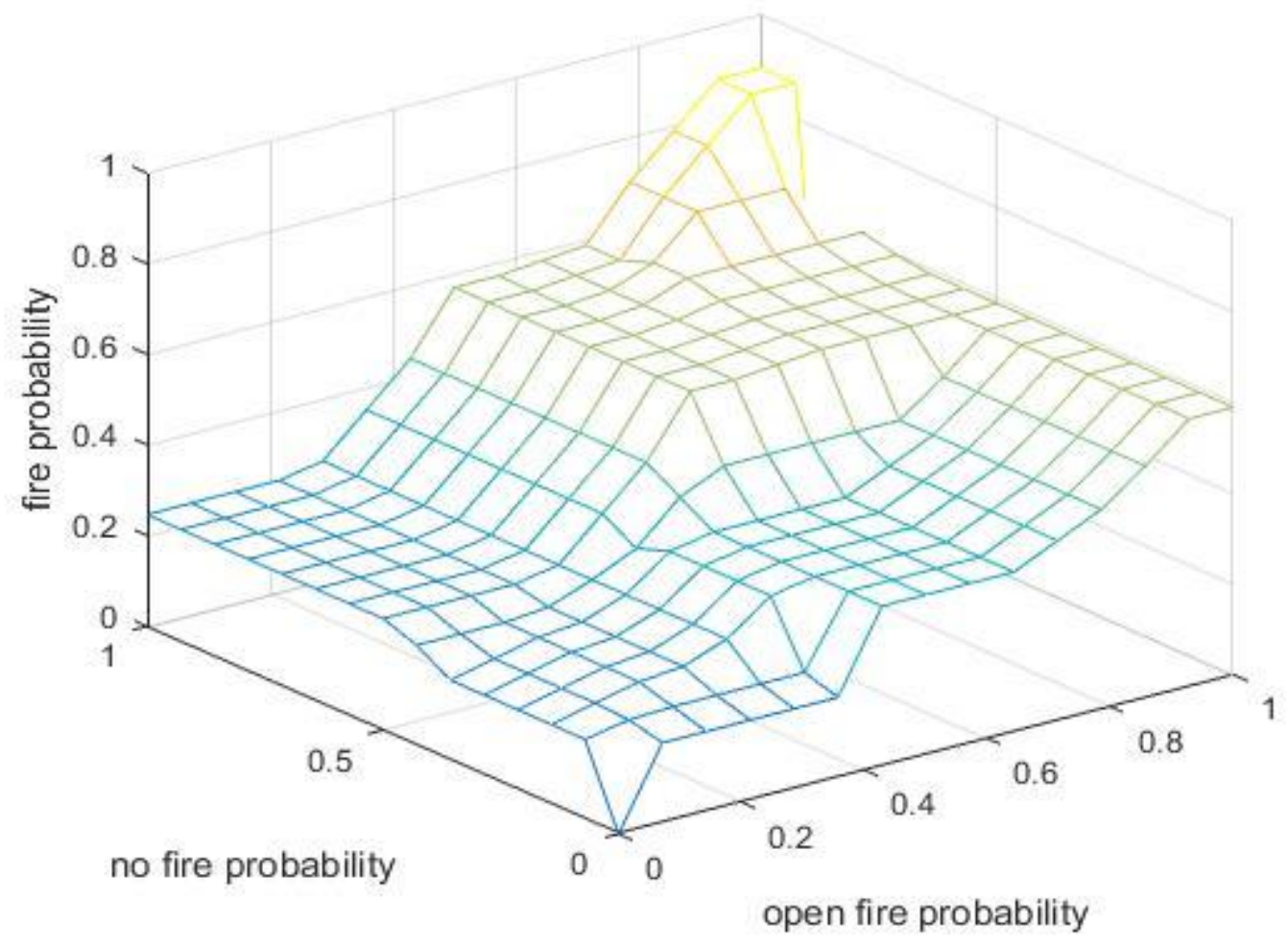

Figure 5. Relationship among open fire probability, no fire probability and fire probability

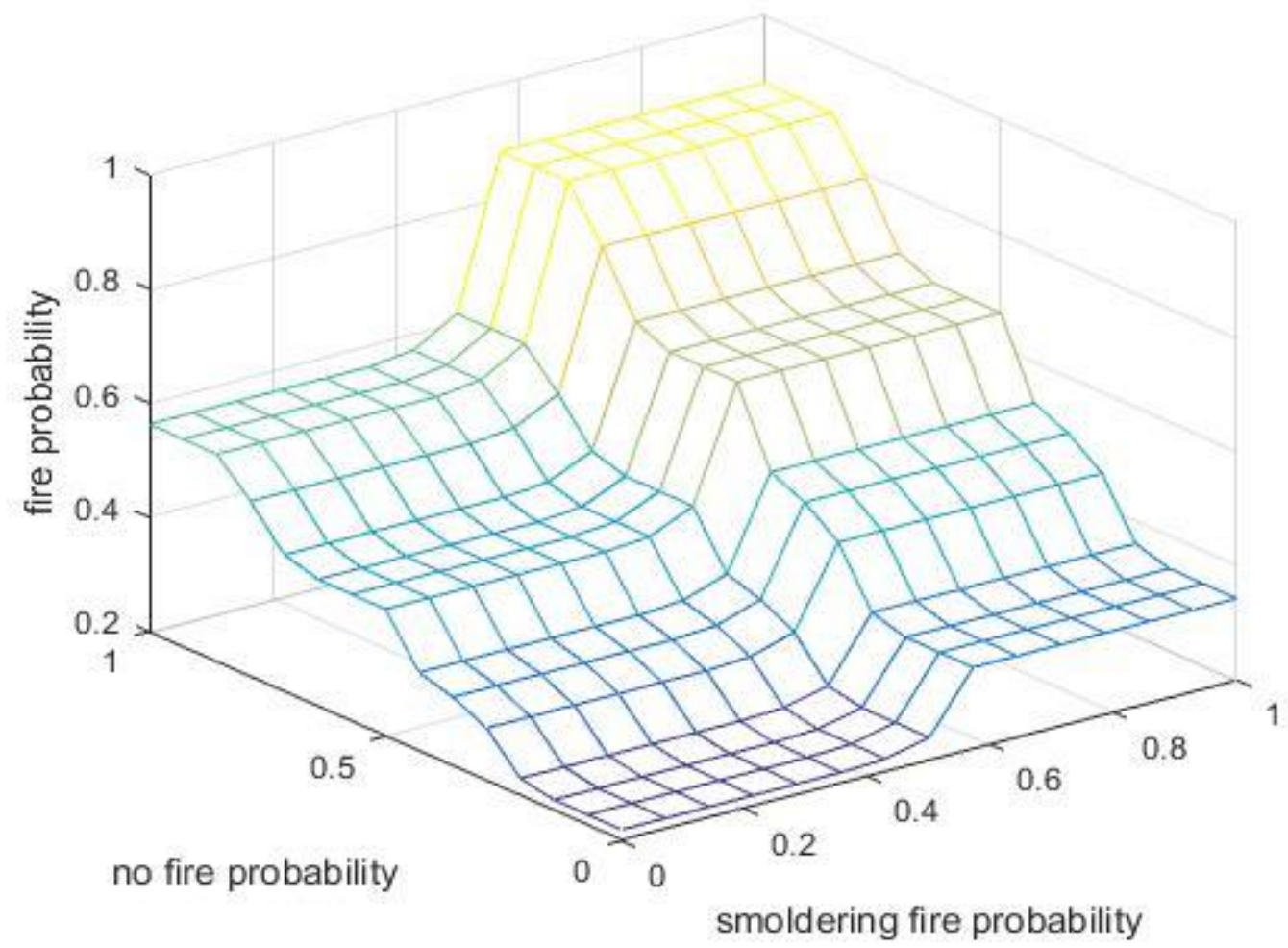

Figure 6. Relationship among smoldering fire probability, no fire probability and fire probability 


\section{Results}

We constructed a fuzzy Bayesian network structure in which Bayesian networks and fuzzy systems are connected in series for forest fire prevention warning. We identified the Bayesian network model for forest fire prevention and designed the fuzzy controller. We use MATLAB to verify the simulation results. The results show that the Bayesian network of the algorithm can accurately give open fire probability, smoldering fire probability and no fire probability. The fuzzy controller can accurately give fire probability. Therefore, the forest fire prevention early warning method based on fuzzy Bayesian network is very effective in forest fire prevention early warning and has broad application prospects.

\section{Acknowledgements}

This work was supported by the Special Funds for the Cultivation of Guangdong College Students' Scientific and Technological Innovation (PDJH2018B0062).

\section{References}

Cai, Q. S., \& Chen, X. R. (2018). Bayesian network structure fusion algorithm based on score function. Computer Engineering and Applications. http://kns.cnki.net/kcms/detail/11.2127.TP.20181017.1659.010.html

Cheng, J. Z., Zhu, W., Fang, W., Wang, C., Sun, J., \& Xiong, Z. (2018). Fault Diagnosis of Hydropower Unit Based on Fuzzy Cognitive Bayesian Network Model. Hydropower Energy Science, 36(216), 141-144.

Gao, H. Y. (2018). Skyline-based three-dimensional forest fire simulation analysis system. Surveying and spatial geographic information, 41(6), 108-110.

Ge, H. Y. (2018). Characteristics of Forest Fires and Countermeasures for Forest Fire Prevention. Modern gardening, 366(18), 227. http://www.cnki.net/kcms/doi/10.14051/j.cnki.xdyy.2018.18.187.html

Gu, J. R. (2000). Application of Fuzzy Control Technology in Analog Fire Alarm System. Atomic Energy Science and Technology, 34(5).

Jensen, F. V. (1996). Bayesian networks basics. AISB quarterly, 9-22.

Lam, W., \& Segre, A. M. (2002). A distributed learning algorithm for Bayesian inference networks. IEEE Transactions on Knowledge and Data Engineering, 14(1), 93-105. https://doi.org/10.1109/69.979975

Li, X. W., Zhao, G, Yu, X. B., \& Yu, Q. (2013). Responses of forest fires to ecological factors in different regions and their probability models. Acta Ecologica Sinica, 33(4), 1219-1229. https://doi.org/10.5846/stxb201205270783

Li, Y. J., Zheng, W., Chen, J., \& Liu, C. (2017). Fire Monitoring and Application Based on Meteorological Satellite. Aerospace Shanghai, 34(4), 62-72. http://www.cnki.net/kcms/doi/10.19328/j.cnki.1006-1630.2017.04.008.html

Liu, C. X., Fan, P., Wang, H. T., Guo, J., \& Ke, R. (2017). Modeling forest fire risk assessment based on BP neural network of transmission line. Power System Protection and Control, 45(17), 100-105. http://www.dlbh.net/dlbh/ch/reader/view_abstract.aspx?doi=10.7667/PSPC161228

Sun, S. J., Zhang, Z. Q., \& Han, H. (2017). Study on the Method of Power Network Operation Early Warning Using Remote Sensor Monitoring and Locating of Satellites Based on Power Grid GIS. Electric Power, 50(4), 181-184. http://www.chinapower.org/CN/10.11930/j.issn.1004-9649.2017.04.181.04

Wang, D., Zhou, A. M., Cong, J. H., \& Zhao, M. S. (2017). Forest-fire prevention management system design based on big data processing. Journal of Central South University of Forestry \& Technology, 37(11), 30-37. http://www.cnki.net/kcms/doi/10.14067/j.cnki.1673-923x.2017.11.006.html

Zhang, C., \& Yang, H. Q. (2017). Study on Fire Positioning Scheme and Fire Alarm Warning Based on Infrared Technology. Measurement \& Control Technology, 36(7), 33-37. http://www.cnki.net/kcms/doi/10.19708/j.ckjs.2017.07.009.html

Zhao, Y. Q. (2015). Fire identification algorithm based on fuzzy neural network. Computer Simulation, 32(2), 369-373.

\section{Copyrights}

Copyright for this article is retained by the author(s), with first publication rights granted to the journal.

This is an open-access article distributed under the terms and conditions of the Creative Commons Attribution license which permits unrestricted use, distribution, and reproduction in any medium, provided the original work is properly cited. 